\title{
FINITE SYMMETRIC TRILINEAR INTEGRAL TRANSFORM OF DISTRIBUTIONS. PART II
}

\author{
G. L. WAGHMARE AND S. V. MORE
}

Received 30 December 2004; Revised 12 April 2006; Accepted 25 April 2006

The finite symmetric trilinear integral transform is extended to distributions by using quite different technique than Zemanian (1968) and Dube (1976) and an inversion formula is established using Parseval's identity. The operational calculus generated is applied to find the temperature inside an equilateral prism of semi-infinite length.

Copyright (c) 2006 Hindawi Publishing Corporation. All rights reserved.

\section{Introduction}

Sen [6] with the help of trilinear coordinates has solved different types of boundary value problems relating to boundaries in the form of an equilateral triangle.

An equilateral triangular region $T$ is described by the set

$$
\left\{x=\left(x_{1}, x_{2}, x_{3}\right) / 0<x_{i}<p, x_{1}+x_{2}+x_{3}=p, x_{i} \in \mathbb{R}, i=1,2,3\right\},
$$

where $x_{1}, x_{2}$, and $x_{3}$ are the trilinear coordinates of a point and $p$ is the height of an equilateral triangle.

Sen [6] has also expressed two-dimensional Laplace operator in trilinear coordinates as

$$
\begin{aligned}
\nabla_{1}^{2} & \equiv \frac{\partial^{2}}{\partial x^{2}}+\frac{\partial^{2}}{\partial y^{2}} \\
& \equiv \frac{\partial^{2}}{\partial x_{1}^{2}}+\frac{\partial^{2}}{\partial x_{2}^{2}}+\frac{\partial^{2}}{\partial x_{3}^{2}}-\frac{\partial^{2}}{\partial x_{1} \partial x_{2}}-\frac{\partial^{2}}{\partial x_{2} \partial x_{3}}-\frac{\partial^{2}}{\partial x_{1} \partial x_{3}} \equiv \square \quad \text { (say) }
\end{aligned}
$$

Later Patil [4] has developed the symmetric integral transform of function of trilinear coordinates which is defined on $T$ as

$$
S(f)(n)=F(n)=\int_{0}^{p} \int_{0}^{p} \int_{0}^{p} f\left(x_{1}, x_{2}, x_{3}\right) \psi_{n}\left(x_{1}, x_{2}, x_{3}\right) d x_{1} d x_{2} d x_{3},
$$


2 Finite symmetric trilinear integral transform

where $\psi_{n}\left(x_{1}, x_{2}, x_{3}\right)=\sin \lambda_{n} x_{1}+\sin \lambda_{n} x_{2}+\sin \lambda_{n} x_{3}$ are eigenfunctions corresponding to the eigenvalues $\lambda_{n}=2 n \pi / p, n=1,2,3, \ldots$, in an eigenvalue problem

$$
\square \psi_{n}+\lambda_{n}^{2} \psi_{n}=0
$$

subjected to the Dirichlet type of boundary conditions

$$
\psi_{n}(x)=0 \quad \text { at } x_{1}=0, x_{2}=0, x_{3}=0 .
$$

If $f\left(x_{1}, x_{2}, x_{3}\right)$ is continuous, has piecewise continuous first- and second-order partial derivatives on $T$, and satisfies the above Dirichlet type of boundary conditions, then inverse transform of (1.6) is given by

$$
f\left(x_{1}, x_{2}, x_{3}\right)=\sum_{n=1}^{\infty} F(n) c_{n} \psi_{n}\left(x_{1}, x_{2}, x_{3}\right),
$$

where $1 / c_{n}=\int_{0}^{p} \int_{0}^{p} \int_{0}^{p} \psi_{n}^{2} d x_{1} d x_{2} d x_{3}=3 p^{3} / 2$ (see [4, page 129]).

In this paper we extend the finite symmetric trilinear integral transform to distributions analogous to the method employed in [1] which is quite different than Zemanian [8] and Dube [2] and establish an inversion theorem by using Parseval's identity as in [3]. At the end we find the temperature inside an equilateral prism of semi-infinite length.

\section{The testing function space $A$}

Let $A$ denote the set of all infinitely differentiable complex-valued functions $\phi$ defined on $T$ which satisfy the following two conditions:

(i) $\square^{m} \phi$ satisfy Dirichlet type of boundary conditions on $T$ for each $m=0,1,2, \ldots$;

(ii)

$$
\alpha_{m}(\phi)=\sup _{x \in T}\left|\square^{m} \phi(x)\right|<\infty \quad \text { for each } m=0,1,2, \ldots
$$

We note that $A$ is nonempty and for each $n \in \mathbb{N}$, eigenfunction $\psi_{n}(x)$ is in $A$.

$A$ is a linear space. The topology of $A$ is that generated by the countable multinorm $\left\{\alpha_{m}\right\}_{m=0}^{\infty}$.

Theorem 2.1. A is complete and therefore a Fréchet space.

The proof of this theorem is similar to the proof of [7, Theorem 3.1].

For every $\phi \in A$, the finite symmetric trilinear integral transform

$$
S(\phi)(n)=\int_{0}^{p} \int_{0}^{p} \int_{0}^{p} \phi(x) \psi_{n}(x) d x_{1} d x_{2} d x_{3}
$$

exists and by (1.6), one has

$$
\phi(x)=\sum_{n=1}^{\infty} c_{n} S(\phi)(n) \psi_{n}(x) .
$$


We call the sequence $(S(\phi)(n))_{n \in \mathbb{N}}$ as the finite symmetric trilinear integral transform $S(\phi)$ of $\phi$. Therefore

$$
S(\phi)=(S(\phi)(n))_{n \in \mathbb{N}} \cdot
$$

The expression (2.3) can be seen as an inversion formula for the said transform.

Proposition 2.2. The map $\phi \rightarrow S(\phi)$ is a continuous linear transformation from $A$ into $l^{\infty}$. Proof. It can be proved by making use of the property of integrals and

$$
\|S(\phi)\|_{l^{\infty}}=\sup _{n}|S(\phi)(n)| \leq 3 p^{3} \alpha_{0}(\phi) .
$$

Let $L_{2}(T)$ denote the set of complex-valued functions $\phi$ defined on $T$ such that

$$
\|\phi\|=\left[\int_{0}^{p} \int_{0}^{p} \int_{0}^{p}|\phi(x)|^{2} d x_{1} d x_{2} d x_{3}\right]^{1 / 2}<\infty .
$$

An inner product in $L_{2}(T)$ is defined by

$$
(\phi, \psi)=\int_{0}^{p} \int_{0}^{p} \int_{0}^{p} \phi(x) \overline{\psi(x)} d x_{1} d x_{2} d x_{3}, \quad \phi, \psi \in L_{2}(T),
$$

where $\overline{\psi(x)}$ denotes the complex conjugate of $\psi(x)$.

We prove the following results which we need in subsequent sections.

Proposition 2.3. If $\phi \in A$, then

$$
S\left(\square^{m} \phi\right)(n)=(-1)^{m} \lambda_{n}^{2 m} S(\phi)(n) \quad \text { for every } n \in \mathbb{N}, m=0,1,2, \ldots
$$

Proof. Integrating by parts and using boundary conditions, we get

$$
S(\square \phi)(n)=\int_{0}^{p} \int_{0}^{p} \int_{0}^{p} \phi(x) \square \psi_{n}(x) d x_{1} d x_{2} d x_{3}=(-1) \lambda_{n}^{2} S(\phi)(n) \quad \text { for every } n \in \mathbb{N} .
$$

Using (2.9), it is quite simple to see that

$$
S\left(\square^{m} \phi\right)(n)=(-1)^{m} \lambda_{n}^{2 m} S(\phi)(n) \quad \text { for every } n \in \mathbb{N}, m=0,1,2, \ldots
$$

Proposition 2.4. Let $\phi \in A$. Then the series

$$
\sum_{n=1}^{\infty} c_{n}\left|S\left(\square^{m} \phi\right)(n)\right|^{2}
$$

converges and Bessel's inequality

$$
\sum_{n=1}^{\infty} c_{n}\left|S\left(\square^{m} \phi\right)(n)\right|^{2} \leq\left\|\square^{m} \phi\right\|^{2}<\infty
$$

holds for each $m=0,1,2,3, \ldots$. 
4 Finite symmetric trilinear integral transform

Proof. Expanding the inner product and using the fact that $\left\{\psi_{n}\right\}$ is an orthogonal set, we get

$$
0 \leq\left\|\square^{m} \phi-\sum_{k=1}^{n} c_{k} S\left(\square^{m} \phi\right)(k) \psi_{k}\right\|^{2}=\left\|\square^{m} \phi\right\|^{2}-\sum_{k=1}^{n} c_{k}\left|S\left(\square^{m} \phi\right)(k)\right|^{2}
$$

and so, for any $n \in \mathbb{N}$,

$$
\sum_{k=1}^{n} c_{k}\left|S\left(\square^{m} \phi\right)(k)\right|^{2} \leq\left\|\square^{m} \phi\right\|^{2}
$$

It is clear that the series (2.11) converges and taking the limit as $n \rightarrow \infty$ we get (2.12).

Proposition 2.5. If $\phi \in A$, then the series

$$
\sum_{n=1}^{\infty}(-1)^{m} c_{n} \lambda_{n}^{2 m} S(\phi)(n) \psi_{n}(x), \quad m=0,1,2, \ldots,
$$

converges absolutely and uniformly over $T$.

Proof. We have

$$
\left|(-1)^{m} c_{n} \lambda_{n}^{2 m} S(\phi)(n) \psi_{n}(x)\right| \leq 3 c_{n} \lambda_{n}^{2 m}|S(\phi)(n)| \quad(x \in T, n=1,2, \ldots) .
$$

By using Proposition 2.3, Cauchy-Schwarz inequality, and (2.12), we get

$$
\sum_{n=1}^{k} 3 c_{n} \lambda_{n}^{2 m}|S(\phi)(n)| \leq 3 c_{0}\left\|\square^{m+1} \phi\right\| \quad \text { for every } k \in \mathbb{N}, \text { where } c_{0}=\left[\sum_{n=1}^{\infty} \frac{c_{n}}{\lambda_{n}^{4}}\right]^{1 / 2} .
$$

In view of $c_{n}=2 / 3 p^{3}$ (see [4, page 129]), $\lambda_{n}=2 n \pi / p$, and $\sum_{n=1}^{\infty}\left(1 / n^{4}\right)=\pi^{4} / 90$ (see [3, page 112]), we have $c_{0}=(1 / 6) \sqrt{p / 60}$.

It is obvious that $\sum_{n=1}^{\infty} 3 c_{n} \lambda_{n}^{2 m}|S(\phi)(n)|$ is convergent. By Weierstrass $M$ test, the series (2.15) converges absolutely and uniformly over $T$.

Corollary 2.6. If $\phi \in A$, then

$$
\square^{m} \phi(x)=\sum_{n=1}^{\infty}(-1)^{m} c_{n} \lambda_{n}^{2 m} S(\phi)(n) \psi_{n}(x), \quad m=0,1,2, \ldots,
$$

and the series converges uniformly over $T$.

Proof. From (1.6), we have

$$
\begin{gathered}
\phi(x)=\lim _{n \rightarrow \infty} \sum_{k=1}^{n} c_{k} S(\phi)(k) \psi_{k}(x), \\
\square^{m} \phi(x)=\lim _{n \rightarrow \infty} \rrbracket^{m}\left(\sum_{k=1}^{n} c_{k} S(\phi)(k) \psi_{k}(x)\right)=\lim _{n \rightarrow \infty} \sum_{k=1}^{n} c_{k}(-1)^{m} \lambda_{k}^{2 m} S(\phi)(k) \psi_{k}(x) .
\end{gathered}
$$


In view of (2.19) and in view of Proposition 2.5, it is inferred that the series $\sum_{n=1}^{\infty}(-1)^{m} c_{n} \lambda_{n}^{2 m} S(\phi) \cdot(n) \psi_{n}(x)$ converges to $\square^{m} \phi$ uniformly on $T$ for each $m=0,1,2, \ldots$

The following is an immediate consequence of Corollary 2.6.

Corollary 2.7. For all $\phi \in A, \phi_{n} \rightarrow \phi$ in $A$, where $\phi_{n}(x)=\sum_{k=1}^{n} c_{k} S(\phi)(k) \psi_{k}(x)$.

Theorem 2.8. For every $\phi \in A$, Parseval's identity holds, that is,

$$
\sum_{n=1}^{\infty} c_{n}|S(\phi)(n)|^{2}=\|\phi\|^{2}
$$

Equivalently

$$
\left(S\left(\phi_{1}\right), S\left(\phi_{2}\right)\right)=\sum_{n=1}^{\infty} c_{n} S\left(\phi_{1}\right)(n) \overline{S\left(\phi_{2}\right)(n)}=\left(\phi_{1}, \phi_{2}\right) .
$$

Proof. We have from Proposition 2.4 that

$$
\left\|\phi-t_{n}\right\|^{2}=\|\phi\|^{2}-\sum_{k=1}^{n} c_{k}|S(\phi)(k)|^{2}, \quad \text { where } t_{n}(x)=\sum_{k=1}^{n} c_{k} S(\phi)(k) \psi_{k}(x) .
$$

But

$$
\left\|\phi-t_{n}\right\|^{2}=\int_{0}^{p} \int_{0}^{p} \int_{0}^{p}\left|\phi(x)-t_{n}(x)\right|^{2} d x_{1} d x_{2} d x_{3} \leq\left[\alpha_{0}\left(\phi-t_{n}\right)\right]^{2} p^{3} .
$$

Hence

$$
0 \leq\|\phi\|^{2}-\sum_{k=1}^{n} c_{k}|S(\phi)(k)|^{2} \leq\left[\alpha_{0}\left(\phi-t_{n}\right)\right]^{2} p^{3} .
$$

Taking the limit as $n \rightarrow \infty$ and using Corollary 2.7, we get (2.20).

By using polarization identity, we get (2.21).

\section{The space of rapidly decreasing sequences}

Let $B$ be the set of all complex sequences $\left(a_{n}\right)_{n \in \mathbb{N}}$ satisfying

$$
\sum_{n=1}^{\infty} c_{n} \lambda_{n}^{2 m}\left|a_{n}\right|<\infty \quad \forall m=0,1,2, \ldots
$$

$B$ is a linear space and

$$
\beta_{m}\left(\left(a_{n}\right)_{n \in \mathbb{N}}\right)=\sum_{n=1}^{\infty} c_{n} \lambda_{n}^{2 m}\left|a_{n}\right|, \quad m=0,1,2, \ldots
$$

defines a countable multinorm on $B . B$ is complete and therefore a Fréchet space. 
6 Finite symmetric trilinear integral transform

Theorem 3.1. For each continuous linear functional $h$ defined on $B$, there exist a positive constant $C$ and a nonnegative integer $r$ such that for every $\left(a_{n}\right)_{n \in \mathbb{N}} \in B$,

$$
\left|\left\langle h,\left(a_{n}\right)_{n \in \mathbb{N}}\right\rangle\right| \leq C_{0 \leq k \leq r}^{\max } \beta_{k}\left(\left(a_{n}\right)_{n \in \mathbb{N}}\right) .
$$

The proof is similar to the proof of [8, Theorem 1.8.1].

THEOREM 3.2. The finite symmetric trilinear integral transform $S$ is a homeomorphism from $A$ onto the space $B$.

Proof. If $\phi \in A$, then, by Proposition $2.5, \sum_{n=1}^{\infty} c_{n} \lambda_{n}^{2 m}|S(\phi)(n)|<\infty$ for every $m=0,1,2, \ldots$

It is simple to prove that the mapping $S: A \rightarrow B$ defined by $S(\phi)=(S(\phi)(n))_{n \in \mathbb{N}}$ is one-one and onto.

Hence $S^{-1}: B \rightarrow A$ exists and is given by

$$
S^{-1}(a)(x)=S^{-1}\left(\left(a_{n}\right)_{n \in \mathbb{N}}\right)(x)=\sum_{n=1}^{\infty} c_{n} a_{n} \psi_{n}(x), \quad x \in T, \text { for each } a=\left(a_{n}\right)_{n \in \mathbb{N}} \in B .
$$

Assume that $\left(\phi_{k}\right)_{k \in \mathbb{N}} \rightarrow \phi$ in $A$ as $k \rightarrow \infty$. By Proposition 2.3,

$$
\begin{aligned}
& \lambda_{n}^{2 m} \mid S\left(\phi_{k}\right)(n)-S(\phi)(n) \mid \\
&=\left|S\left(\square^{m}\left(\phi_{k}-\phi\right)\right)(n)\right| \\
& \leq 3 p^{3} \alpha_{m}\left(\phi_{k}-\phi\right) \longrightarrow 0 \quad \text { as } k \longrightarrow \infty, \forall n \in \mathbb{N}, m=0,1,2, \ldots, \\
& \beta_{m}\left(S\left(\phi_{k}\right)-S(\phi)\right) \\
& \quad=\sum_{n=1}^{\infty} c_{n} \lambda_{n}^{2 m}\left|S\left(\phi_{k}\right)(n)-S(\phi)(n)\right| \longrightarrow 0 \quad \text { as } k \longrightarrow \infty, \text { for each } m=0,1,2, \ldots
\end{aligned}
$$

This proves $S$ is continuous. To prove $S^{-1}$ is continuous, we proceed as follows.

Let $a^{k} \rightarrow a$ in $B$ as $k \rightarrow \infty$, where $a^{k}=\left(a_{n}^{(k)}\right)_{n \in \mathbb{N}}, a=\left(a_{n}\right)_{n \in \mathbb{N}}$. Using (3.4),

$$
\begin{gathered}
S^{-1}\left(a^{k}-a\right)(x)=\sum_{n=1}^{\infty} c_{n}\left(a_{n}^{(k)}-a_{n}\right) \psi_{n}(x), \quad x \in T, \\
\alpha_{m}\left(S^{-1}\left(a^{k}-a\right)\right)=\sup _{x \in T}\left|\sum_{n=1}^{\infty} c_{n}\left(a_{n}^{(k)}-a_{n}\right)(-1)^{m} \lambda_{n}^{2 m} \psi_{n}(x)\right| \quad \text { (by Corollary 2.6) } \\
\leq 3 \sum_{n=1}^{\infty} c_{n} \lambda_{n}^{2 m}\left|a_{n}^{(k)}-a_{n}\right| \longrightarrow 0 \quad \text { as } k \longrightarrow \infty, \text { for each } m=0,1,2, \ldots
\end{gathered}
$$

This completes the proof. 


\section{Distribution space}

In this section we will introduce the space of distributions and study its basic properties.

Definition 4.1. A linear functional $U$ on a Fréchet space $A, U: A \rightarrow C$, is called a distribution if there exists a sequence $\left(\chi_{n}\right)_{n \in \mathbb{N}}$ in $A$ such that

$$
\langle U, \phi\rangle=\lim _{n \rightarrow \infty} \int_{0}^{p} \int_{0}^{p} \int_{0}^{p} \chi_{n}(x) \phi(x) d x_{1} d x_{2} d x_{3} \quad \text { exists for each } \phi \in A
$$

The set of all distributions is a complex linear space and it will be denoted by $A^{\prime}$.

Let $A_{0}$ denote the set of all functions $f(x)$ which are continuous, have piecewise continuous first- and second-order partial derivatives on $T$, and satisfy the Dirichlet type of boundary conditions on $T$.

Proposition 4.2. Let $f \in A_{0}$. Then the formula

$$
\left\langle U_{f}, \phi\right\rangle=\int_{0}^{p} \int_{0}^{p} \int_{0}^{p} f(x) \phi(x) d x_{1} d x_{2} d x_{3}, \quad \phi \in A,
$$

defines a distribution $U_{f}$ on $A . A_{0}$ can be embedded in $A^{\prime}$.

Proof. $U_{f}$ is clearly linear. Define $\chi_{n}(x)=\sum_{k=1}^{n} c_{k} S(f)(k) \psi_{k}(x), \chi_{n} \in A$, for all values of $n$.

Moreover, $\chi_{n} \rightarrow f$ uniformly on $T$ follows from Corollary 2.6. Therefore

$$
\left\langle U_{f}, \phi\right\rangle=\lim _{n \rightarrow \infty} \int_{0}^{p} \int_{0}^{p} \int_{0}^{p} x_{n}(x) \phi(x) d x_{1} d x_{2} d x_{3}
$$

which proves that (4.2) defines a distribution $U_{f}$ on $A$.

It is clear that the map $f \rightarrow U_{f}$ is linear, one-to-one, and continuous.

Finally, if $U_{f_{n}} \rightarrow U_{f}$ in image of $A_{0}$, then

$$
\int_{0}^{p} \int_{0}^{p} \int_{0}^{p}\left(f_{n}-f\right)(x) \phi(x) d x_{1} d x_{2} d x_{3}=\left\langle U_{f_{n}}-U_{f}, \phi\right\rangle \longrightarrow 0
$$

as $n \rightarrow \infty$ for all $\phi \in A$.

This implies $f_{n} \rightarrow f$ as $n \rightarrow \infty$. Thus $A_{0}$ can be embedded in $A^{\prime}$.

There are distributions that do not have the form (4.2) with $f \in A_{0}$.

Example 4.3. Dirac function $\delta_{x}$ centered at $x \in T$ is given by

$$
\left\langle\delta_{x}, \phi\right\rangle=\phi(x), \quad \phi \in A .
$$

It is easy to prove that $\delta_{x}$ is linear. 
8 Finite symmetric trilinear integral transform

Take $\chi_{n}(y)=\sum_{k=1}^{n} c_{k} \psi_{k}(x) \psi_{k}(y), x, y \in T$, and $x$ is fixed. Then $\chi_{n}$ is in $A$ for each $n \in \mathbb{N}$,

$$
\lim _{n \rightarrow \infty} \int_{0}^{p} \int_{0}^{p} \int_{0}^{p} x_{n}(y) \phi(y) d y_{1} d y_{2} d y_{3}=\lim _{n \rightarrow \infty} \sum_{k=1}^{n} c_{k} S(\phi)(k) \psi_{k}(x)=\phi(x)=\left\langle\delta_{x}, \phi\right\rangle .
$$

So $\delta_{x} \in A^{\prime}$.

Define $d(\phi, \psi)=\sum_{m=0}^{\infty}\left(1 / 2^{m}\right)\left(\alpha_{m}(\phi-\psi) /\left(1+\alpha_{m}(\phi-\psi)\right)\right)$. Then $d$ is a compatible translation invariant metric on $A[5$, page 27]. Furthermore $(A, d)$ is a complete metric space.

TheOREM 4.4. Every distribution is a continuous linear functional on A.

The proof is similar to the proof of [3, Theorem 3.143].

Proposition 4.5. $A^{\prime}$ is the dual of $A$, that is, $A^{\prime}$ is precisely the collection of all continuous linear functionals from $A$ into $\mathbb{C}$.

Proof. Let $f: A \rightarrow \mathbb{C}$ be a continuous linear functional. For each $\phi \in A$, by Corollary 2.7,

$$
E_{n}(\phi)=\sum_{k=1}^{n} c_{k} S(\phi)(k) \psi_{k} \longrightarrow \phi \quad \text { in } A
$$

We have

$$
\langle f, \phi\rangle=\lim _{n \rightarrow \infty}\left\langle f, E_{n}(\phi)\right\rangle=\lim _{n \rightarrow \infty} \int_{0}^{p} \int_{0}^{p} \int_{0}^{p} \phi(x) \chi_{n}(x) d x_{1} d x_{2} d x_{3}
$$

Thus the condition in the definition of distribution is satisfied with

$$
\chi_{n}(x)=\sum_{k=1}^{n} c_{k}\left\langle f, \psi_{k}\right\rangle \psi_{k}(x)
$$

Proposition 4.6. $A$ is a subspace of $A^{\prime}$ and the topology of $A$ is stronger than that induced on it by $A^{\prime}$.

Proof. Let $f \in A$. By Proposition 4.2,

$$
\langle f, \phi\rangle=\int_{0}^{p} \int_{0}^{p} \int_{0}^{p} f(x) \phi(x) d x_{1} d x_{2} d x_{3}
$$

defines a distribution $U_{f}$. Therefore $A \subset A^{\prime}$.

Further

$$
\xi_{\phi}(f)=|\langle f, \phi\rangle| \leq \alpha_{0}(f) \alpha_{0}(\phi) p^{3} \quad f \in A, \phi \in A,
$$

implies the second statement. 
Proposition 4.7. For each $f \in A^{\prime}$, there exist a nonnegative integer $r$ and a positive constant $C$ such that

$$
|\langle f, \phi\rangle| \leq C_{0 \leq m \leq r}^{\max } \alpha_{m}(\phi)
$$

Here $C$ and $r$ depend on $f$ but not on $\phi$.

The proof is similar to proof of [8, Theorem 1.8.1].

\section{Generalized finite symmetric trilinear integral transform}

The generalized finite symmetric trilinear integral transform $S^{\prime}$ of $f \in A^{\prime}$ is defined by

$$
\left\langle S^{\prime}(f),\left(a_{n}\right)_{n \in \mathbb{N}}\right\rangle=\left\langle f(x), \sum_{n=1}^{\infty} c_{n} a_{n} \psi_{n}(x)\right\rangle, \quad\left(a_{n}\right)_{n \in \mathbb{N}} \in B .
$$

If $\phi(x)=\sum_{n=1}^{\infty} c_{n} a_{n} \psi_{n}(x)$, then, by Theorem 3.2, $\left(a_{n}\right)_{n \in \mathbb{N}}=(S(\phi)(n))_{n \in \mathbb{N}}=S(\phi)$ and (5.1) can be written as

$$
\left\langle S^{\prime}(f), S(\phi)\right\rangle=\langle f, \phi\rangle
$$

Theorem 5.1. $S^{\prime}$ is a homeomorphism from $B^{\prime}$ onto the space $A^{\prime}$.

Proof. This result can be seen as a consequence of Theorem 3.2 and [8, Theorem 1.10.2].

Proposition 5.2. The finite symmetric trilinear integral transform $S$ is a special case of the generalized transform $S^{\prime}$. That is, $S^{\prime} f=S f$ for every $f \in A$.

Proof. By using Proposition 4.6, (5.1), Theorems 3.2, and 2.8, it is simple to prove

$$
\left\langle S^{\prime} f,\left(a_{n}\right)_{n \in \mathbb{N}}\right\rangle=\sum_{n=1}^{\infty} c_{n} S(f)(n) a_{n}=\left\langle(S(f)(n))_{n \in \mathbb{N}},\left(a_{n}\right)_{n \in \mathbb{N}}\right\rangle, \quad\left(a_{n}\right)_{n \in \mathbb{N}} \in B .
$$

Motivated by the above result, we define the generalized integral transform $S^{\prime} f$ of $f \in A^{\prime}$ as

$$
S^{\prime}(f)=\left(\left\langle f(x), \psi_{n}(x)\right\rangle\right)_{n \in \mathbb{N}}
$$

and we set

$$
S^{\prime}(f)(n)=\left\langle f(x), \psi_{n}(x)\right\rangle, \quad \psi_{n} \in A, n \in \mathbb{N} .
$$

We now state and prove an inversion theorem for the elements of $A^{\prime}$ that can be seen as an inversion formula for the $S^{\prime}$-transformation. 
10 Finite symmetric trilinear integral transform

Theorem 5.3. Let $f \in A^{\prime}$. Then

$$
f=\lim _{n \rightarrow \infty} \sum_{k=1}^{n} c_{k}\left\langle f, \psi_{k}\right\rangle \psi_{k}
$$

where the limit is taken in the sense of $A^{\prime}$.

Proof. Let $F_{n}(x)=\sum_{k=1}^{n} c_{k}\left\langle f, \psi_{k}\right\rangle \psi_{k}$. Since $F_{n} \in A$ for every $n$, by Proposition 4.2,

$$
\left\langle F_{n}, \psi_{m}\right\rangle= \begin{cases}\left\langle f, \psi_{m}\right\rangle & \text { if } m \leq n \\ 0 & \text { if } m>n\end{cases}
$$

By using Theorem 2.8, Parseval's identity, we have

$$
\begin{aligned}
\left\langle F_{n}, \phi\right\rangle & =\sum_{k=1}^{\infty} c_{k}\left\langle F_{n}, \psi_{k}\right\rangle S(\phi)(k) \\
& =\sum_{k=1}^{n} c_{k}\left\langle f, \psi_{k}\right\rangle S(\phi)(k) \quad(\text { by }(5.7)) \\
& =\left\langle f, E_{n}(\phi)\right\rangle \quad \text { for every } \phi \in A, E_{n}(\phi)=\sum_{k=1}^{n} c_{k} S(\phi)(k) \psi_{k} .
\end{aligned}
$$

By Corollary 2.7, $E_{n}(\phi) \rightarrow \phi$ for all $\phi \in A$. Therefore

$$
\lim _{n \rightarrow \infty}\left\langle F_{n}, \phi\right\rangle=\lim _{n \rightarrow \infty}\left\langle f, E_{n}(\phi)\right\rangle=\langle f, \phi\rangle, \quad \forall \phi \in A .
$$

Theorem 5.4 (uniqueness theorem). If $f, g \in A^{\prime}$ are such that $S^{\prime}(f)(n)=S^{\prime}(g)(n)$ for every $n$, then $f=g$ in the sense of equality in $A^{\prime}$.

The following example illustrates the inversion theorem.

Example 5.5. Dirac function $\delta_{x}$ centered at $x \in T$ is given by

$$
\left\langle\delta_{x}, \phi\right\rangle=\phi(x), \quad \phi \in A .
$$

In Example 4.3, we have shown that $\delta_{x}$ is in $A^{\prime}$. The finite symmetric trilinear integral transform of $\delta_{x}$ is given as

$$
S^{\prime}\left(\delta_{x}\right)(n)=\left\langle\delta_{x}(t), \psi_{n}(t)\right\rangle=\psi_{n}(x)
$$

By virtue of Proposition 4.2, for all $\phi(t) \in A$,

$$
\left\langle\sum_{m=0}^{\mathbb{N}} c_{m} \psi_{m}(x) \psi_{m}(t), \phi(t)\right\rangle=\sum_{m=0}^{\mathbb{N}} c_{m} S(\phi)(m) \psi_{m}(x) \longrightarrow \phi(x) \quad \text { as } \mathbb{N} \longrightarrow \infty .
$$

But $\phi(x)=\left\langle\delta_{x}(t), \phi(t)\right\rangle$. Therefore $\delta_{x}(t)=\lim _{\mathbb{N} \rightarrow \infty} \sum_{m=0}^{\mathbb{N}} c_{m} \psi_{m}(x) \psi_{m}(t)$. 
A trivial consequence of Theorem 5.3 is the following version of Parseval's identity. Corollary 5.6. Let $\phi \in A$ and $f \in A^{\prime}$. Then

$$
\langle f, \phi\rangle=\sum_{n=1}^{\infty} c_{n}\left\langle f, \psi_{n}\right\rangle S(\phi)(n)=\left\langle S^{\prime} f, S \phi\right\rangle .
$$

\section{Characterization of distributions in $A^{\prime}$ and their generalized finite symmetric trilinear integral transform $S^{\prime}$}

Proposition 6.1. Let $H: B \rightarrow \mathbb{C}$. Then $H \in B^{\prime}$ if and only if there exists a complex sequence $\left(b_{n}\right)_{n \in \mathbb{N}}$ such that

$$
\left|b_{n}\right| \leq C \lambda_{n}^{2 k}, \quad n \in \mathbb{N}
$$

for some $C>0$ and some $k \in \mathbb{N}$, for which

$$
\left\langle H,\left(a_{n}\right)_{n \in \mathbb{N}}\right\rangle=\sum_{n=1}^{\infty} c_{n} a_{n} b_{n}, \quad\left(a_{n}\right)_{n \in \mathbb{N}} \in B .
$$

Proof. Assume that $H$ takes the form (6.2) where $\left(b_{n}\right)_{n \in \mathbb{N}}$ satisfies (6.1). By using (3.2) it is simple to prove $H \in B^{\prime}$. Conversely, let $H \in B^{\prime}$. Then by Theorem 3.1, there exists $k \in \mathbb{N}$ such that

$$
\left|\left\langle H,\left(a_{n}\right)_{n \in \mathbb{N}}\right\rangle\right| \leq C_{0 \leq m \leq k}^{\max } \sum_{n=1}^{\infty} c_{n} \lambda_{n}^{2 m}\left|a_{n}\right| \leq C \sum_{n=1}^{\infty} c_{n} \lambda_{n}^{2 k}\left|a_{n}\right|, \quad\left(a_{n}\right)_{n \in \mathbb{N}} \in B .
$$

We now define the mapping, $J: B \rightarrow J(B) \subset l_{1}$

$$
\left(a_{n}\right)_{n \in \mathbb{N}} \longrightarrow\left(\lambda_{n}^{2 k} a_{n}\right)_{n \in \mathbb{N}}
$$

$J$ is one-one.

Moreover, the linear mapping $M: J(B) \subset l_{1} \rightarrow \mathbb{C}$

$$
\left(\lambda_{n}^{2 k} a_{n}\right)_{n \in \mathbb{N}} \longrightarrow\left\langle H,\left(a_{n}\right)_{n \in \mathbb{N}}\right\rangle
$$

is continuous by virtue of (6.3).

By Hahn-Banach theorem, $M$ can be extended to $l_{1}$ as a member of $l_{1}^{\prime}$. Then there exists $\left(d_{n}\right)_{n \in \mathbb{N}} \in l_{\infty}$ such that

$$
M\left(\lambda_{n}^{2 k} a_{n}\right)_{n \in \mathbb{N}}=\left\langle H,\left(a_{n}\right)_{n \in \mathbb{N}}\right\rangle=\sum_{n=1}^{\infty} d_{n} a_{n} \lambda_{n}^{2 k}, \quad\left(a_{n}\right)_{n \in \mathbb{N}} \in B .
$$

Taking $b_{n}=d_{n} \lambda_{n}^{2 k} / c_{n}$, where $c_{n}=2 / 3 p^{3}\left[4\right.$, page 129], $n \in \mathbb{N}$. Since every sequence in $l_{\infty}$ is bounded sequence, we have

$$
\left\langle H,\left(a_{n}\right)_{n \in \mathbb{N}}\right\rangle=\sum_{n=1}^{\infty} c_{n} a_{n} b_{n} .
$$


12 Finite symmetric trilinear integral transform

Theorem 6.2. Let $\left(b_{n}\right)_{n \in \mathbb{N}}$ be a complex sequence. There exists $f \in A^{\prime}$ such that $F(n)=$ $S^{\prime}(f)(n)=b_{n}, n \in \mathbb{N}$, if and only if there exist $C>0$ and $k \in \mathbb{N}$ such that

$$
\left|b_{n}\right| \leq C \lambda_{n}^{2 k}, \quad n \in \mathbb{N}
$$

Proof. The condition is necessary and follows from Proposition 4.7.

Let $\left(b_{n}\right)_{n \in \mathbb{N}}$ be a sequence satisfying condition (6.8).

For any $\phi \in A$,

$$
\lim _{k \rightarrow \infty}\left\langle\sum_{n=1}^{k} c_{n} b_{n} \psi_{n}(x), \phi(x)\right\rangle \equiv \sum_{n=1}^{\infty} c_{n} b_{n} S(\phi)(n) .
$$

From (6.8) and Theorem 3.2, it is clear that the series $\sum_{n=1}^{\infty} c_{n} b_{n} S(\phi)(n)$ is absolutely convergent.

Define $f: A \rightarrow \mathbb{C}$ by the formula

$$
\langle f, \phi\rangle=\sum_{n=1}^{\infty} c_{n} b_{n} S(\phi)(n), \quad \phi \in A .
$$

Then $f$ is a linear functional on $A$ and

$$
\langle f, \phi\rangle=\lim _{n \rightarrow \infty} \int_{0}^{p} \int_{0}^{p} \int_{0}^{p} \phi(x) \chi_{n}(x) d x_{1} d x_{2} d x_{3}
$$

where $\chi_{n}(x)=\sum_{k=1}^{n} c_{k} b_{k} \psi_{k}(x) \in A$ for every $n \in \mathbb{N}$.

By using orthogonal relations, we have $S^{\prime}(f)(n)=b_{n}, n \in \mathbb{N}$.

Thus the condition is sufficient.

\section{Operational calculus}

Integrating by parts and using boundary conditions, one can easily prove that if $f \in A_{0}$, then

$$
\langle\square f, \phi\rangle=\langle f, \llbracket \phi\rangle \quad \text { for every } \phi \in A \text {. }
$$

It allows us to define that for any $f \in A^{\prime}$,

$$
\langle\square f, \phi\rangle=\langle f, \llbracket \phi\rangle, \quad \phi \in A .
$$

It is clear that $\square f \in A^{\prime}$.

It can also be seen inductively that for any integer $m$,

$$
\left\langle\square^{m} f, \phi\right\rangle=\left\langle f, \square^{m} \phi\right\rangle \quad \text { for every } \phi \in A
$$

and $\square^{m} f \in A^{\prime}$.

Therefore

$$
\left\langle\square^{m} f, \psi_{n}\right\rangle=\left\langle f, \square^{m} \psi_{n}\right\rangle=(-1)^{m} \lambda_{n}^{2 m}\left\langle f, \psi_{n}\right\rangle .
$$


That is,

$$
S^{\prime}\left(\square^{m} f\right)(n)=(-1)^{m} \lambda_{n}^{2 m} S^{\prime}(f)(n)
$$

which gives an operation transform formula.

Now consider the partial differential equation of the form

$$
G(\square) f=h,
$$

where given $h$ and unknown $f$ are required to be in $A^{\prime}$, and $G$ is a polynomial such that $G\left(-\lambda_{n}^{2}\right) \neq 0, n=1,2,3, \ldots$

By applying the operation transform formula (7.5) to (7.6), we obtain

$$
\begin{gathered}
G\left(-\lambda_{n}^{2}\right) F(n)=H(n), \quad F(n)=\left(S^{\prime} f\right)(n), H(n)=\left(S^{\prime} h\right)(n), \\
F(n)=\frac{H(n)}{G\left(-\lambda_{n}^{2}\right)} .
\end{gathered}
$$

By applying the inversion theorem (Theorem 5.3), we get

$$
f=\sum_{n=1}^{\infty} c_{n} \frac{H(n)}{G\left(-\lambda_{n}^{2}\right)} \psi_{n}, \quad G\left(-\lambda_{n}^{2}\right) \neq 0, \text { for } n=1,2, \ldots
$$

\section{Application}

In this section we apply the present theory to find the temperature inside an equilateral prism of semi-infinite length. The formulation of the problem is given below.

Find the conventional function $v(x, z)$ on the domain

$$
R \equiv\left\{(x, z)=\left(x_{1}, x_{2}, x_{3}, z\right) / 0<x_{i}<p, i=1,2,3, x_{1}+x_{2}+x_{3}=p, 0<z<\infty\right\}
$$

that satisfies Laplace equation

$$
\frac{\partial^{2} v}{\partial x_{1}^{2}}+\frac{\partial^{2} v}{\partial x_{2}^{2}}+\frac{\partial^{2} v}{\partial x_{3}^{2}}-\frac{\partial^{2} v}{\partial x_{1} \partial x_{2}}-\frac{\partial^{2} v}{\partial x_{1} \partial x_{3}}-\frac{\partial^{2} v}{\partial x_{2} \partial x_{3}}+\frac{\partial^{2} v}{\partial z^{2}}=0
$$

in $R$ and the following boundary conditions:

(i) as $z \rightarrow 0+, v(x, z) \rightarrow f(x) \in A^{\prime}$ in the sense of convergence in $A^{\prime}$;

(ii) as $x_{i} \rightarrow 0+, i=1,2,3, v(x, z)$, converges to zero uniformly on $Z \leq z<\infty$ for each $Z>0$;

(iii) as $z \rightarrow \infty, v(x, z)$ converges uniformly to zero uniformly on $0<x_{i}<p, i=1,2,3$. Every section of the prism by a plane perpendicular to $z$-axis is an equilateral triangle with its centroid on the $z$-axis,

$$
\text { set } V(n, z)=S^{\prime}(v(x, z))=\left\langle v, \psi_{n}\right\rangle \text {. }
$$


14 Finite symmetric trilinear integral transform

By applying the finite symmetric trilinear integral transform $S^{\prime}$ to $(8.2)$, we arrive at

$$
-\lambda_{n}^{2} V(n, z)+\frac{\partial^{2}}{\partial z^{2}} V(n, z)=0
$$

whose general solution is

$$
V(n, z)=A(n) e^{\lambda_{n} z}+B(n) e^{-\lambda_{n} z}, \quad A(n) \text { and } B(n) \text { do not depend on } z
$$

In view of boundary condition (iii), it is reasonable to choose $A(n)=0$ and $B(n)=F(n)$ because of boundary condition (i). Therefore,

$$
V(n, z)=F(n) e^{-\lambda_{n} z}
$$

Applying the inversion theorem (Theorem 5.3) to the above equation, we get

$$
v(x, z)=\sum_{n=1}^{\infty} c_{n} F(n) e^{-\lambda_{n} z} \psi_{n}(x) .
$$

We now verify that (8.7) is truly a solution of (8.2).

By Theorem 6.2, there exist $C>0$ and $k \in \mathbb{N}$ such that $|F(n)| \leq C \lambda_{n}^{2 k}$. For $Z \leq z<\infty$ where $Z>0$, the $n$th term of the series (8.7) satisfies the condition

$$
\left|c_{n} F(n) e^{-\lambda_{n} z} \psi_{n}(x)\right| \leq 3 c_{n} C \lambda_{n}^{2 k} e^{-\lambda_{n} Z} .
$$

Using $c_{n}=2 / 3 p^{3}, \lambda_{n}=2 n \pi / p$, and $e^{-\lambda_{n} z}<(2 k+2) ! / \lambda_{n}^{2 k+2} Z^{2 k+2}$, we get

$$
\left|c_{n} F(n) e^{-\lambda_{n} z} \psi_{n}(x)\right|<C^{*} \frac{1}{n^{2}}, \quad \text { where } C^{*}<\frac{(2 k+2) ! C}{2 \pi^{2} P \cdot Z^{2 k+2}}
$$

By Weierstrass $M$ test, the series on the right-hand side of (8.7) converges absolutely and uniformly over $R$. The factor $e^{-\lambda_{n} z}$ ensures the uniform convergence of any series obtained by term-by-term differentiation of (8.7) with respect to $x_{i}, i=1,2,3$, or $z$. We may apply the operator $\square+D_{z}^{2}$ under the summation sign in (8.7). Since $e^{-\lambda_{n} z} \psi_{n}\left(x_{1}, x_{2}, x_{3}\right)$ satisfies Laplace equation, so does $v$. Thus the differential equation (8.2) is satisfied in the conventional sense.

To verify the boundary condition (i), we have to show that for each $\phi \in A$,

$$
\langle v(x, z), \phi(x)\rangle \longrightarrow\langle f, \phi\rangle \quad \text { as } z \longrightarrow 0+.
$$


Now, for any fixed $z>0$, the series (8.7) defines a function in $A_{0}$, and by Proposition 4.2 we have for every $\phi \in A$,

$$
\begin{aligned}
\langle v, \phi\rangle & =\int_{0}^{p} \int_{0}^{p} \int_{0}^{p}\left[\sum_{n=1}^{\infty} c_{n} F(n) e^{-\lambda_{n} z} \psi_{n}(x)\right] \phi(x) d x_{1} d x_{2} d x_{3} \\
& =\sum_{n=1}^{\infty} c_{n} F(n) e^{-\lambda_{n} z} S(\phi)(n) .
\end{aligned}
$$

The series in (8.11) converges uniformly for all $z>0$. By taking the limit as $z \rightarrow 0+$, one has

$$
\begin{aligned}
\lim _{z \rightarrow 0+}\langle v, \phi\rangle & =\sum_{n=1}^{\infty} c_{n} F(n) S(\phi)(n) \\
& =\lim _{\mathbb{N} \rightarrow \infty}\left\langle\sum_{n=1}^{\mathbb{N}} c_{n} F(n) \psi_{n}(x), \phi(x)\right\rangle \\
& =\langle f, \phi\rangle \quad \text { by virtue of Theorem 5.3. }
\end{aligned}
$$

Finally, for $Z \leq z \leq \infty(Z>0)$, we have from (8.7) that

$$
|v(x, z)| \leq \sum_{n=1}^{\infty} c_{n}|F(n)| e^{-\lambda_{n} Z}\left|\psi_{n}(x)\right| .
$$

The series in (8.13) converges absolutely and uniformly on T. So we may take limit as $x_{i} \rightarrow 0+$ under the summation sign in (8.13), which verifies boundary condition (ii).

In the same way we have

$$
|v(x, z)| \leq 3 \sum_{n=1}^{\infty} c_{n}|F(n)| e^{-\lambda_{n} z} .
$$

The series in (8.14) converges uniformly on $0<z<\infty$. By taking the limit as $z \rightarrow \infty$ under the summation sign in (8.14), one verifies boundary condition (iii).

\section{Acknowledgments}

The authors express their sincere thanks to the referees for valuable suggestions.

\section{References}

[1] J. J. Betancor, M. Linares, and J. M. R. Méndez, A distributional convolution for a generalized finite Fourier transformation, Proceedings of the American Mathematical Society 128 (2000), no. $2,547-556$.

[2] L. S. Dube, On finite Hankel transformation of generalized functions, Pacific Journal of Mathematics 62 (1976), no. 2, 365-378.

[3] R. J. Iorio Jr. and V. M. Iorio, Fourier Analysis and Partial Differential Equations, Cambridge Studies in Advanced Mathematics, vol. 70, Cambridge University Press, Cambridge, 2001. 


\section{Finite symmetric trilinear integral transform}

[4] P. B. Patil, Methods of mathematical physics and their applications, Ph.D. thesis, Marathwada University (M.S.), Aurangabad, 1975.

[5] W. Rudin, Functional Analysis, Tata McGraw-Hill, New Delhi, 1976.

[6] B. Sen, Trilinear co-ordinates and boundary value problems, Bulletin of the Calcutta Mathematical Society 60 (1968), no. 1-2, 25-30.

[7] G. L. Waghmare and S. V. More, On finite symmetric trilinear integral transform of distributions, Journal of Indian Academy of Mathematics 26 (2004), no. 1, 137-166.

[8] A. H. Zemanian, Generalized Integral Transformations, John Wiley \& Sons, New York, 1968.

G. L. Waghmare: Department of Mathematics, Institute of Science, Nagpur-440 001, MS, India E-mail address: glwaghmare@rediff.com

S. V. More: Department of Mathematics, Institute of Science, Nagpur-440 001, MS, India E-mail address: glwagmare@rediffmail.com 


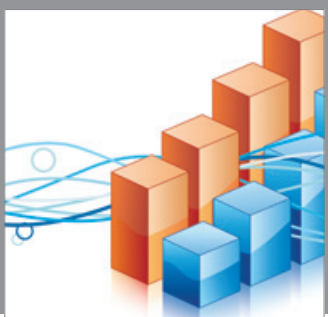

Advances in

Operations Research

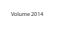

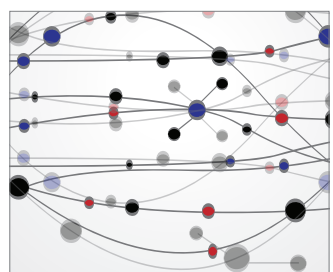

\section{The Scientific} World Journal
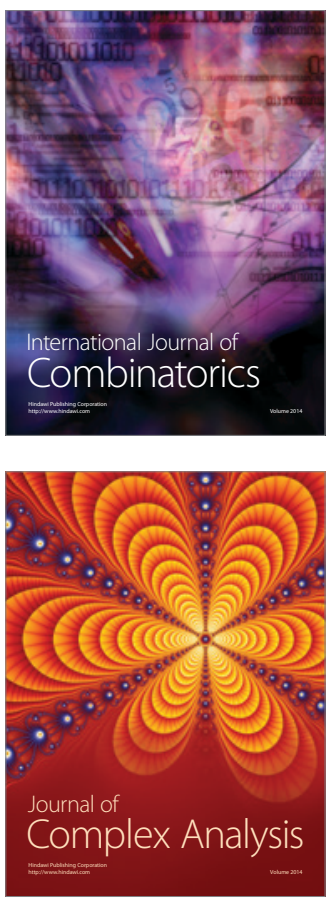

International Journal of

Mathematics and

Mathematical

Sciences
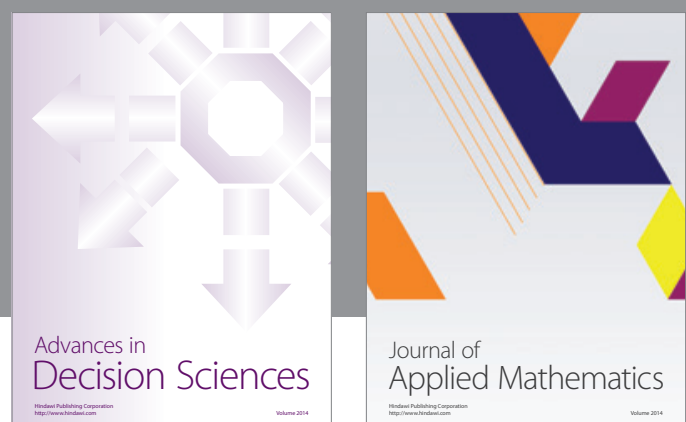

Journal of

Applied Mathematics
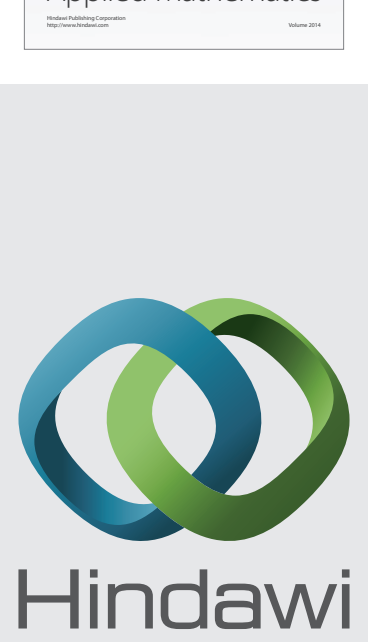

Submit your manuscripts at http://www.hindawi.com
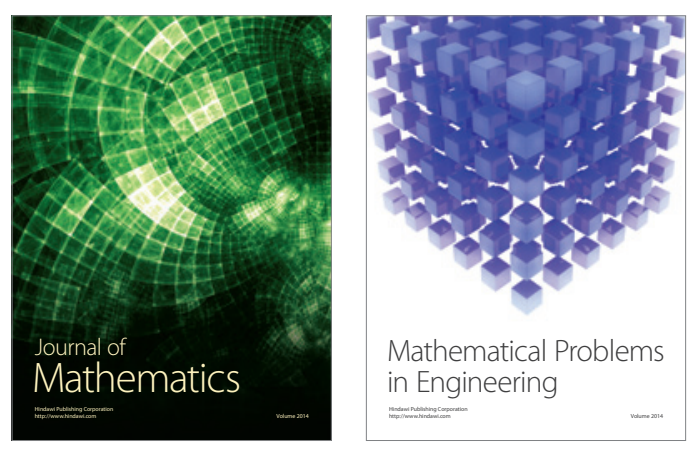

Mathematical Problems in Engineering
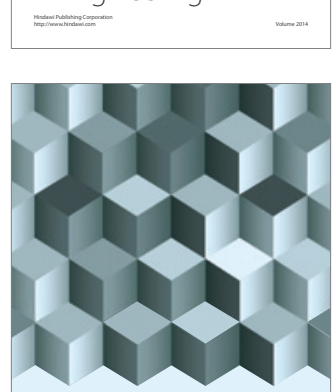

Journal of

Function Spaces
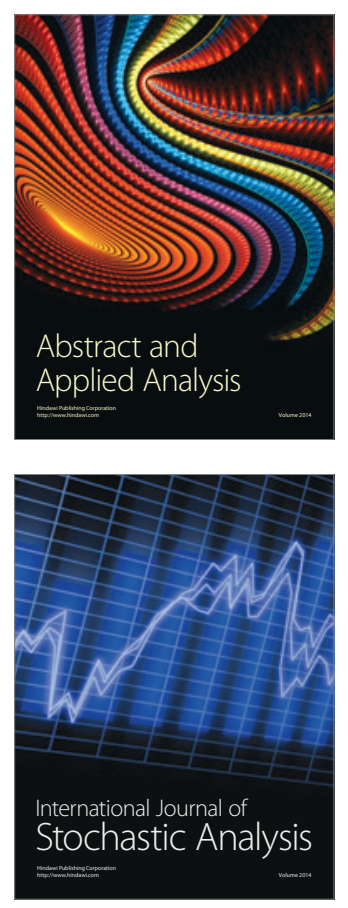

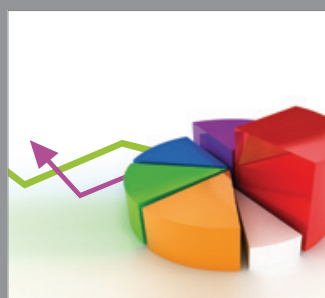

ournal of

Probability and Statistics

Promensencen
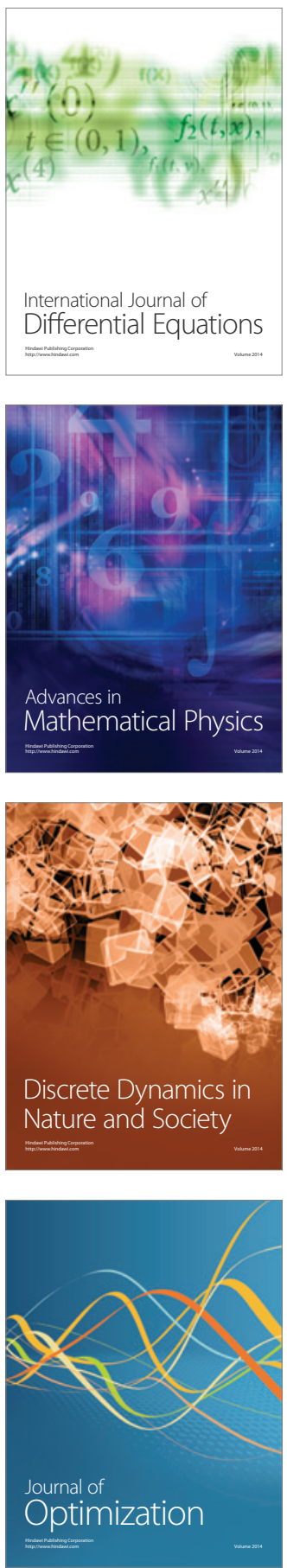